\title{
Contratualização na Atenção Primária à Saúde: a experiência de Portugal e Brasil
}

\author{
Contractual arrangements in Primary Health Care: \\ the experience of Portugal and Brazil
}

Vanessa Costa e Silva ${ }^{1}$

Ana Escoval ${ }^{2}$

Virginia Alonso Hortale ${ }^{1}$
${ }^{1}$ Escola Nacional de Saúde Pública, Fiocruz. R. Leopoldo Bulhões 1480, Manguinhos. 22.400-00 Rio de Janeiro RJ Brasil. vanessa@ensp.fiocruz.br

${ }^{2}$ Escola Nacional de Saúde Pública, Universidade Nova de Lisboa.

\begin{abstract}
The contractual arrangements adopted in the Family Health Strategy are a topic as yet scantly addressed in studies. It is introduced in Brazil in different models in accordance with the contracting entity and the legal status of the contracted service provider; and in Portugal, it is based on a model of inter-governmental contractual arrangements with the Family Health Units. In this paper, the current status of contractual arrangements in both countries is presented and their attributes of joint planning, accountability with autonomy and performance-based incentive programs are discussed. The main contributions are: better coordination of the health organizations; substitution of the hierarchical command-control logic with greater participation; and accountability of professionals with enhanced performance of their activities. The conclusion reached is that one of the facilitating elements is gradual construction, in an environment of ongoing learning, with health professionals playing the leading role. However, the major challenges include transparency in the implementation of processes, the enforcement of sound auditing mechanisms and information systems, as well as the continuous review of indicators and their suitability for the health needs of the population. Key words Primary health care, Family health, Health management, Contractual arrangements, Contracts
\end{abstract}

Resumo A contratualização desenvolvida na Estratégia Saúde da Família é um tema ainda pouco estudado. É introduzida no Brasil em diferentes modelos, de acordo com o ente federativo contratante e o estatuto jurídico do prestador de serviços contratado; e em Portugal, a partir de modelo único de contratualização público-público com as Unidades de Saúde Familiar. Apresenta-se neste artigo o panorama atual da contratualização nos dois países, por meio de estudo de casos, e discutem-se seus atributos de planejamento conjunto, responsabilização com autonomia e sistema de incentivos ao desempenho. As principais contribuições da contratualização são: melhor coordenação das organizações de saúde; substituição da lógica hierárquica de comando-controle pela de maior participação; e responsabilização dos profissionais com melhor desempenho das suas atividades. Conclui-se que um dos elementos facilitadores é sua construção gradual, em um ambiente de aprendizagem permanente, com o protagonismo dos profissionais de saúde; e que seus maiores desafios são a transparência na condução dos processos, $o$ fortalecimento dos mecanismos de auditoria e dos sistemas de informação, bem como a revisão permanente dos indicadores de monitoramento e sua adequação às necessidades de saúde da população. Palavras-chave Atenção primária, Saúde da família, Gestão em saúde, Contratualização, Contratos 


\section{Introdução}

Desde a década de oitenta, vários países realizam reformas administrativas, políticas ou econômicas. Da perspectiva dos modelos de gestão de serviços públicos, há um grupo de estratégias que incluem: a tentativa de redução do déficit fiscal, mediante a expansão da economia de mercado e privatização desses serviços; a busca pela qualidade e reorientação voltada para a necessidade dos cidadãos; a descentralização dos processos políticos e administrativos; e uma maior participação do setor privado e não governamental na prestação desses serviços.

Tal movimento foi influenciado pelos fundamentos da Nova Gestão Pública (NGP) ${ }^{1-3}$, que a partir da emergência dos temas "crise e reforma do Estado" nos anos 80, nos Estados Unidos e Inglaterra, difunde-se para os países da Organização para a Cooperação e Desenvolvimento Econômico. Corresponde a um conjunto de argumentos e filosofias administrativas proposto para a gestão pública, e baseia-se na Teoria Agente-Principal ${ }^{4,5}$, bem como incorpora aspectos da gestão empresarial: reengenharia, grupos de interesses (stakeholders), foco em resultados, flexibilização, remuneração vinculada ao desempenho, entre outros.

Segundo um dos principais ideólogos da $\mathrm{NGP}^{6}$, ela é, antes de tudo, um espaço de debate sobre a administração pública e um diálogo profissional entre a comunidade científica e os agentes públicos. E como tal, deveria ser descartada a tentação de acepções formais dirigindo o debate para a busca de respostas, adaptadas a cada circunstância, às questões de como gerenciar e controlar os sistemas burocráticos públicos.

Estudos sobre esse tema ${ }^{7,8}$ mostram que não há um padrão, mas sim diversos modelos adaptados ao contexto social, econômico e político de cada país. Ou seja, não se trataria de uma disputa hegemônica, mas sim de uma tendência generalizada de incorporação de uma orientação gerencialista em diferentes contextos político-institucionais.

No âmbito dos sistemas de saúde, adota-se em países como Inglaterra, Suécia, Itália, Espanha, França e Portugal, a separação pelo Estado das funções de financiamento e prestação de serviços. Essa separação pode ser institucional, quando o prestador é privado, com ou sem fins lucrativos; ou organizacional, quando o prestador é público, e implica na introdução de mecanismos de contratualização entre as partes, os quais definem a função dos vários atores e a partilha de riscos e responsabilidades'. Seu objetivo seria o de melhorar a coordenação e o desempenho dos serviços e aumentar a transparência na prestação de contas dos resultados alcançados ${ }^{10}$.

Para analisar um processo de contratualização, há que se ter em conta as singularidades das organizações de saúde, que demandam exigências específicas de coordenação e gestão. Elas são consideradas "organizações profissionais", pois dependem dos profissionais de saúde para seu bom funcionamento. O mecanismo dominante de coordenação é a padronização das qualificações, saber e habilidades, decidida pelas entidades de classe, o que produz tensões entre o poder técnico, concentrado na base da organização (núcleo operacional), e o poder administrativo da direçãa ${ }^{11}$.

Entende-se a utilização de contratos entre financiadores e prestadores como um exercício de planejamento compartilhado, articulação, acordos negociados e convergência de interesses. Nessa perspectiva, seria um mecanismo de coordenação alternativo aos modelos hierarquizados, e que objetiva o aumento da responsabilização de gestores e trabalhadores quanto aos resultados, em um conjunto de práticas que intensifiquem a gestão por compromissos e contribuam para a efetividade dos serviços de saúde ${ }^{12}$.

Assume-se o pressuposto que a contratualização e, especificamente, seus atributos de planejamento compartilhado; responsabilização com autonomia; e sistema de incentivos ao bom desempenho dos profissionais, poderiam contribuir de forma positiva na coordenação das organizações de saúde e, consequentemente, na melhoria de seu desempenho.

Assim, este estudo apresenta aspectos teóricos da contratualização e sua aplicação prática em experiências distintas no âmbito da atenção primária à saúde (APS) em Portugal e no Brasil, bem como discute as práticas que facilitam ou dificultam tais processos, com vistas a uma melhor compreensão do tema e à produção de outras narrativas.

O fortalecimento da APS é uma importante estratégia de reordenamento dos sistemas de saúde, por sua comprovada eficiência na utilização dos recursos e efetividade das práticas, especialmente quando estruturadas para se dar como primeiro contato, abordagem integral e coordenação do usuário nos diferentes serviços ${ }^{13}$. A "saúde da família", foco deste estudo, reorienta o modelo assistencial da APS, ao preconizar a abordagem familiar e comunitária e o trabalho em equipe, e deu novo impulso às Reformas Sanitárias iniciadas na década de oitenta em vários países. Entretanto, a heterogeneidade de suas práticas, bem como dos modelos de gestão adaptados a cada realidade, sugerem a difusão e análise de experiências locais 
que visem à ampliação do acesso da população a este nível de atenção, bem como a melhoria da qualidade do cuidado em saúde.

\section{Método}

Trata-se de um estudo de casos que tem como foco os serviços de APS que utilizam a abordagem familiar no Brasil, no Sistema Único de Saúde (SUS) e em Portugal, no Serviço Nacional de Saúde (SNS). Este país foi escolhido porque instituiu a contratualização como dispositivo de gestão desde a implantação das Unidades de Saúde Familiar (USF). Considerando a diversidade da experiência brasileira, fruto das autonomias legislativas e matizes políticas de cada governo, optou-se pela análise de alguns aspectos relevantes nos processos e referidos a duas estratégias de contratualização. A primeira, na administração direta - Secretaria Municipal de Saúde da cidade de Curitiba; a segunda, no setor privado não lucrativo - Organizações Sociais de Saúde da cidade do Rio de Janeiro.

A discussão levou em consideração as características demográficas, a organização do Sistema de Saúde e, em especial, as possíveis diferenças entre os dois países e as singularidades do desenvolvimento histórico-cultural dos sistemas de proteção social em cada país.

As informações foram obtidas em dois momentos. O primeiro, por meio de levantamento bibliográfico nas bases PubMed, Web of Sciences, Scielo, Repositório Científico de Acesso Aberto de Portugal, Index e B-On e também no acervo da Escola Nacional de Saúde Pública da Universidade Nova de Lisboa, e pela identificação dos principais documentos sobre o assunto. O segundo, por meio de entrevistas com informantes-chave no contexto português.

Considerando que os dois países adotam práticas diferenciadas na incorporação da contratualização, construiu-se uma matriz (Quadro 1) que relaciona as características gerais da APS (organização, regulação e saúde familiar), bem como os três atributos considerados nesse estudo como relevantes ao processo de contratualização no que diz respeito à sua contribuição como um mecanismo de coordenação participativo e de ajustamento de interesses entre gestores e profissionais de saúde e, portanto, mais adequado às singularidades das organizações de saúde.

\section{As unidades de saúde da família e os modelos de contratualização}

\section{A experiência portuguesa}

A constituição das USF fez parte de uma ampla reforma, voltada para a APS, iniciada em Portugal em 2005. Baseia-se na auto-organização de equipes formadas por médicos especialistas em medicina geral e familiar, enfermeiros e apoio administrativo que seguem os princípios de orientação comunitária, flexibilidade organizacional e de gestão, autonomia e responsabilização, melhoria contínua da qualidade, contratualização e avaliação ${ }^{14}$.

Sua implementação é crescente: em 2006 havia 43 USF em funcionamento; em 2010, 242 e, em outubro de 2013, 346 unidades, com uma cobertura potencial de $41 \%$ da população total estimada em 10.562.178 habitantes ${ }^{15}$. Toda a gestão, financiamento e regulação é federal, por intermédio das Administrações Regionais de Saúde (ARS) e Agrupamentos de Centros de Saúde (ACES) ${ }^{16}$.

A contratualização, modelo de gestão instituído, baseou-se em experiências positivas anteriores. No Projeto Alfa ${ }^{17}$, de 1998, alguns centros de saúde foram descentralizados atendendo aos princípios de autonomia e responsabilização. Esse projeto, bem avaliado em termos de melhoria no acesso e na qualidade dos serviços prestados, deu origem ao "Regime Remuneratório Especial" dos médicos que introduziu, pela primeira vez na administração pública portuguesa, a remuneração baseada no desempenho ${ }^{18}$.

A legislação ${ }^{19}$ induz o desenvolvimento institucional das equipes com a assunção gradativa de responsabilização, autonomia e sistema de incentivos ao classificar as USF em três modelos. No modelo A, a USF encontra-se em fase de aprendizagem e experimentação da lógica contratual; no B tem desenvolvimento institucional compatível com níveis mais exigentes de contratualização. Importante destacar que, em ambos os modelos, as USF pertencem à administração pública direta, ou seja, trata-se de uma separação organizacional entre financiador e prestador. Todavia, o modelo $\mathrm{C}$, que se refere às USF dos setores social (organizações não governamentais ou filantrópicas), cooperativo ou privado, não foi implementado.

No final de 2012, e a partir da experiência adquirida de contratualização na administração pública, foi constituído um grupo de trabalho no Ministério da Saúde para analisar as condições de abertura, a título experimental, do modelo C de USF. A justificativa seria suprir, em caráter complementar, as eventuais insuficiências de cobertura e assimetrias de acesso da população a um médico de família ${ }^{20}$. 


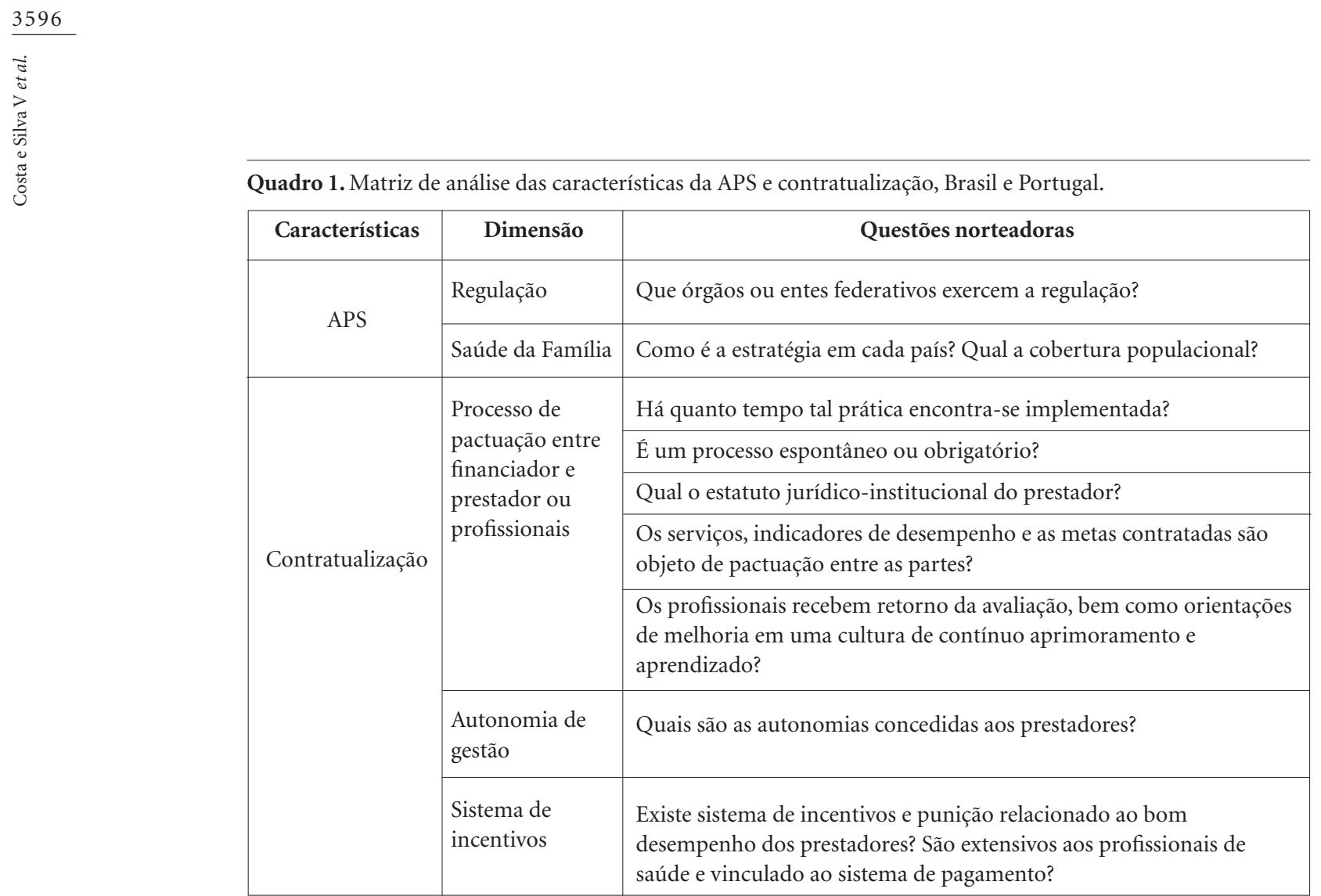

Estudo de caso publicado em $2010^{21}$, em que se realizaram entrevistas e técnicas de grupo nominal com especialistas, mostrou que a construção e o aperfeiçoamento gradativo desse processo, respaldado por experiências anteriores bem avaliadas e com o protagonismo dos médicos de família, contribuíram de forma positiva para a contratualização na APS.

\section{A experiência brasileira}

A Estratégia Saúde da Família inicia em 1994, com experiências-piloto em alguns municípios, e hoje, com o acúmulo técnico e político dos três níveis de gestão do SUS e os resultados positivos nos principais indicadores de saúde das populações assistidas, a adoção da Saúde da Família pelos serviços municipais é a estratégia prioritária de expansão e consolidação da atenção primária no país.

Dados de agosto de $2013^{22}$ revelam a existência de 34.216 equipes de saúde da família (agente comunitário de saúde, técnico ou auxiliar de enfermagem, enfermeiro e médico), das quais $66 \%$ com equipes de saúde bucal (odontólogo, técnico em higiene dental e auxiliar de saúde bucal) e cobertura potencial de 55,8 \% da população em 5.319 municípios brasileiros (95\% do total de municípios). Por lei, os municípios são responsáveis pela decisão de implementação da estratégia, gestão e pela quase totalidade do financiamento, enquanto os governos federal e estadual cuidam da normatização, monitoramento e avaliação e concedem incentivos financeiros para adesão à estratégia.

Nesse contexto, os modelos de contratualização variam em decorrência das particularidades políticas e institucionais de cada governo e localidade, bem como o ente federativo (governo federal, estado ou município) contratante e o estatuto jurídico do prestador de serviços contratado. Identificam-se, no levantamento bibliográfico, quatro principais modelos: (i) entre uma Secretaria Municipal de Saúde -SMS, unidades de saúde e equipes pertencentes à administração direta; (ii) entre uma SMS e o setor privado não lucrativo, denominado Organização Social de Saúde - OSS; (iii) entre uma Fundação Estatal de Saúde da Família (fundação pública de direito privado da administração indireta) e os municípios e equipes; e (iv) entre o Ministério da Saúde, municípios e equipes por meio do Programa de Monitoramento da Qualidade e do Acesso na Atenção Básica (PMAQ-AB). Os dois primeiros são discutidos neste estudo.

A SMS de Curitiba, cidade com 1.764.541habitantes, instituiu há mais de uma década a contratualização com todas as unidades de atenção primária, incluindo as atuais 208 equipes de saúde da família que cobrem potencialmente $40,4 \%$ da população ${ }^{22}$. O processo iniciou-se com ampla 
capacitação de profissionais e dirigentes da SMS, para a revisão do planejamento local e dos protocolos clínicos e a apropriação das informações gerenciais no processo de decisão ${ }^{23}$.

O modelo de contratualização com Organizações Sociais de Saúde (OSS) implica na separação institucional das funções do Estado de financiamento e prestação de serviços. OSS é uma denominação atribuída pelo poder público às organizações do setor privado sem fins lucrativos, que atuam em parceria formal com o Estado, mediante candidatura e cumprimento de exigências legais ${ }^{24,25}$. No governo federal foram regulamentadas em 1998, mas sua expansão ocorreu principalmente na atenção hospitalar e nas esferas estadual e municipal, que utilizaram suas autonomias legislativas e implantaram versões locais.
Entre os municípios, destaca-se o Rio de Janeiro que, desde 2009, estabeleceu o modelo de contratação de OSS como diretriz político-operacional para ampliação da Estratégia Saúde da Família ${ }^{26}$. Com uma população atual de 6.355.949 habitantes, passou de uma cobertura potencial de 6,97\% da população e 124 equipes, em janeiro de 2009, para uma cobertura de $40 \%$ da população e 738 equipes em junho de $2013^{22}$.

As cinco OSS contratadas foram selecionadas em licitação pública e assinaram Contrato de Gestão com a Secretaria Municipal de Saúde, cujo objeto é a operacionalização e apoio às atividades e serviços de saúde da família ${ }^{27}$, no âmbito da região da cidade pré-definida em edital.

São apresentados, a seguir, e sintetizados no Quadro 2 para os casos analisados, os resultados

Quadro 2. Características da contratualização na APS, Portugal, Curitiba e Rio de Janeiro, 2013.

\begin{tabular}{|c|c|c|c|c|}
\hline Características & Questões norteadoras & Portugal & Curitiba & Rio de Janeiro \\
\hline \multirow{5}{*}{$\begin{array}{l}\text { Processo de } \\
\text { pactuação } \\
\text { entre } \\
\text { financiador e } \\
\text { prestador ou } \\
\text { profissionais }\end{array}$} & Ano de implantação & 2006 & 2002 & 2010 \\
\hline & Adesão das equipes & Espontânea & Obrigatória & Obrigatória \\
\hline & $\begin{array}{l}\text { Estatuto jurídico-institucional do } \\
\text { prestador }\end{array}$ & $\begin{array}{l}\text { Público - } \\
\text { administração } \\
\text { direta federal }\end{array}$ & $\begin{array}{l}\text { Público - } \\
\text { administração } \\
\text { direta municipal }\end{array}$ & $\begin{array}{l}\text { Privado sem } \\
\text { fins lucrativos } \\
\text { (OSS) }\end{array}$ \\
\hline & $\begin{array}{l}\text { Os indicadores de desempenho e } \\
\text { as metas contratadas são objeto da } \\
\text { pactuação entre as partes }\end{array}$ & Sim & $\begin{array}{l}\text { Sim, as equipes } \\
\text { escolhem } 13 \\
\text { indicadores de } \\
\text { monitoramento } \\
\text { em uma lista de } \\
74 \text { pré-definidos } \\
\text { pela SMS. }\end{array}$ & $\begin{array}{l}\text { Indicadores } \\
\text { são definidos } \\
\text { pela SMS, } \\
\text { algumas metas } \\
\text { são pactuadas } \\
\text { de acordo com } \\
\text { especificidades } \\
\text { locais. }\end{array}$ \\
\hline & $\begin{array}{l}\text { Os profissionais recebem retorno } \\
\text { da avaliação, bem como orientações } \\
\text { de melhoria em uma cultura } \\
\text { de contínuo aprimoramento e } \\
\text { aprendizado }\end{array}$ & $\begin{array}{l}\text { Sim, } \\
\text { mensalmente }\end{array}$ & $\begin{array}{l}\text { Sim, } \\
\text { trimestralmente }\end{array}$ & $\begin{array}{l}\text { Em início de } \\
\text { implantação }\end{array}$ \\
\hline $\begin{array}{l}\text { Autonomia } \\
\text { de Gestão }\end{array}$ & $\begin{array}{l}\text { Tipo de autonomias concedidas aos } \\
\text { prestadores e profissionais de saúde }\end{array}$ & Organizacional & Organizacional & $\begin{array}{l}\text { Financeira e } \\
\text { Administrativa } \\
\text { parcial }\end{array}$ \\
\hline \multirow{2}{*}{$\begin{array}{l}\text { Sistema de } \\
\text { Incentivos }\end{array}$} & $\begin{array}{l}\text { Existência de sistema de incentivos e } \\
\text { punição ao prestador }\end{array}$ & Sim & Sim & Sim \\
\hline & $\begin{array}{l}\text { Vinculação entre sistema de } \\
\text { incentivos e pagamento dos } \\
\text { profissionais }\end{array}$ & Sim & Sim & Sim \\
\hline
\end{tabular}


referentes à implantação dos atributos relevantes para um adequado processo de contratualização.

\section{Contratos - processo de pactuação, responsabilização e autonomias}

Entre os entes contratantes, a pactuação conjunta dos objetivos, indicadores, metas e mecanismos de monitoramento das ações em saúde visa ao desenvolvimento de uma cultura gerencial de parceria na busca de resultados. Pressupõe que ambas as partes conheçam as necessidades de saúde da população, os objetivos institucionais, os recursos envolvidos, as debilidades presentes e a capacidade instalada. Além disso, tem seu foco no acesso aos cuidados de saúde com a qualidade esperada e assente em um sistema de informação adequado.

Dependendo do grau de maturidade e desenvolvimento institucional dos entes contratantes, os termos do contrato podem ser negociados ou impostos pelo financiador. O desejável é a pactuação bilateral, uma forma de trazer ao processo decisório, como sujeitos de formulação de diretrizes, os indivíduos e grupos antes considerados objetos da ação gerencial. Seria o momento de financiador e prestador assumirem responsabilidades. O primeiro, com a garantia dos recursos e controle dos resultados; o segundo, com a organização do cuidado em saúde e alcance das metas e resultados pactuados.

Nessa perspectiva, a efetiva responsabilização só aconteceria em um ambiente de autonomia do prestador de serviço. Não se trataria de independência, mas de descentralização de poderes, funções e processos, com flexibilidade técnica, organizacional, financeira ou administrativa. As autonomias técnica e organizacional seriam reguladas por um conjunto de instrumentos formais explícitos e elaborados pelos prestadores, como, por exemplo, plano de ação, regulamento interno ou carteira de serviços.

Em Portugal, o Ministério da Saúde desenvolveu em 2006 os indicadores de monitoramento das USF com participação de representantes dos profissionais. Anualmente as USF elaboram seu plano de ação a partir de metodologia definida pelo Ministério da Saúde e assinam com a direção do ACES uma Carta-Compromisso que abrange as dimensões, acessibilidade, desempenho assistencial, satisfação dos usuários e eficiência ${ }^{28}$. As equipes têm autonomia na organização dos processos de trabalho, o que foi considerado fator de motivação e estímulo à sua criatividade e inovação $0^{29}$. Não há autonomia administrativa ou financeira. A aquisição de insumos, contratação e demissão de pessoal e o financiamento são reali- zados pela Administração Regional de Saúde em uma separação dita organizacional das funções de financiamento e prestação dos serviços.

Em Curitiba, o dispositivo de contratualização, chamado Termo de Compromisso de Gestão, constitui-se de pactuações de responsabilidades entre equipes, gestores e representantes dos usuários no Conselho de Saúde. A participação dos últimos promoveria transparência e sua corresponsabilização nas ações planejadas, o que pode ser considerado um diferencial em relação às outras experiências analisadas.

Essas pactuações baseiam-se em prioridades estratégicas definidas previamente no Plano Plurianual, na Agenda Municipal e nas Conferências Municipais de Saúde, nas evidências (protocolos clínicos) e no custo-efetividade das tecnologias de saúde, na disponibilidade e capacidade de ajustar a oferta de serviços, e nas necessidades, demandas e expectativas dos usuários ${ }^{30}$. O contexto de cada unidade de saúde possibilita a definição dos objetivos do Plano Operativo Anual, dispositivo considerado por Azevedo ${ }^{23}$ como um potencializador do planejamento local.

No Contrato de Gestão do município do Rio de Janeiro, identifica-se, na especificação dos serviços, o forte controle e normatização da SMS em relação às OSS. Ela acompanha a produção mínima de procedimentos esperados por cada profissional de saúde, alguns indicadores de boa prática clínica, o número máximo de pessoas cadastradas por equipe, a articulação com outros níveis assistenciais e a implementação das atividades de promoção da saúde e prevenção da doença.

$\mathrm{Na}$ aquisição de insumos, contratação e demissão de pessoal, a autonomia é parcial, pois a SMS normatiza a remuneração dos profissionais bem como o limite financeiro a ser comprometido com recursos humanos, aquisição de equipamentos e contratos de manutenção das unidades de saúde. Sobre esse aspecto, cabe destacar que, para o Direito Administrativo ${ }^{31}$, o contrato de gestão, quando celebrado com organizações sociais, restringe a sua autonomia, pois, entidades privadas terão que sujeitar-se a exigências contidas no contrato de gestão.

Também os indicadores de monitoramento são pré-definidos pela SMS e algumas metas são pactuadas de acordo com especificidades locais. A atuação conjunta de gerentes das OSS e gerentes de cada coordenação regional de saúde (servidores públicos) é uma prática instituída, o que poderia facilitar o acompanhamento, a regulação, por parte da SMS, e induzir as cinco OSS a uma execução mais uniforme das práticas na Estratégia Saúde da Família em todo o município. 
A avaliação trimestral é realizada por uma Comissão Técnica de Acompanhamento, composta por servidores públicos dos níveis central e distrital da SMS. Os indicadores de desempenho estabelecidos são acompanhados com foco nos resultados alcançados. Além disso, é feito o confronto com as metas pactuadas e com a relação custo-benefício no desenvolvimento das atividades. Embora o controle seja voltado para os resultados, as OSS também prestam contas mensais da execução financeira, do patrimônio e dos recursos humanos.

\section{Sistema de incentivos}

A existência de incentivos ao bom desempenho dos prestadores seria um importante dispositivo da contratualização, além de ser, de acordo com alguns estudos ${ }^{32,33}$, uma estratégia para melhorar a qualidade do cuidado em saúde. Há dois principais incentivos: o institucional e o individual. $\mathrm{O}$ primeiro significa a autorização para a unidade de saúde utilizar um recurso determinado na melhoria das condições de trabalho do conjunto das equipes. Isso pode conferir maior autonomia organizacional aos prestadores de serviços, já que são estes que definem como utilizar o recurso. Como exemplos, a capacitação dos profissionais, a melhoria de espaços físicos e a aquisição de equipamentos.

No segundo tipo de incentivo, nas experiências analisadas, o pagamento do profissional é composto por uma parte fixa e outra variável, esta última concedida quando do alcance das metas, individuais ou por equipe, e previamente negociadas. De acordo com Kazandjian ${ }^{34}$, não se trataria de um pagamento por performance $(P 4 P)$, ainda que seja o termo mais utilizado, mas sim de um "pagamento ajustado pelo desempenho", ou seja um ajuste no pagamento existente a partir de aspectos do desempenho medidos e metas alcançadas. Ainda segundo o autor, este seria o modelo mais apropriado dentre os diversos existentes.

A introdução desses incentivos permitiria a disseminação dos objetivos contratualizados entre todos os profissionais, mesmo aqueles que não participam do processo de negociação; o correto preenchimento do sistema de informação, pois sem bons registros clínicos não é possível garantir cuidados adequados e, muito menos avaliar o trabalho realizado e os cuidados prestados; e o monitoramento contínuo do processo de trabalho e busca de estratégias para o alcance das metas pactuadas.

Entretanto, para ser um instrumento de gestão efetivo deveria existir transparência na condução do processo e clareza quanto aos objetivos e critérios de avaliação dos profissionais, além de indicadores e metas mensuráveis e ajustados a cada equipe.

Em Portugal, o pagamento de incentivos varia de acordo com o grau de desenvolvimento institucional das equipes, ou seja; no modelo A pode-se contratar um conjunto adicional de serviços, pagos em horas extras aos profissionais ou em incentivos institucionais às USF; no modelo $\mathrm{B}$ aplica-se, além do incentivo institucional, um regime retributivo especial para todos e que integra o salário-base, os suplementos e a compensação pelo desempenho. Esse procedimento é mensal e inclui a lista de usuários ajustada por idade, número de visitas domiciliares e de hora-profissional trabalhada; as compensações por desempenho, relacionados à pactuação anual de atividades específicas de vigilância aos pacientes vulneráveis ou de alto risco; e um conjunto adicional de serviços, quando previamente negociados ${ }^{35}$.

Estudo realizado por Gouveia et al..$^{36}$ sobre os custos e o sistema de remuneração e incentivos das USF, concluiu que sua utilização foi positiva em relação à eficiência e à despesa pública, o que reforçaria a percepção de que tal dispositivo contribuiria para melhores resultados em saúde.

Em Curitiba, o Programa de Incentivo de Desenvolvimento e Qualidade (IDQ) faz parte do Plano Operativo Anual e compõe-se de uma parte variável atribuída trimestralmente, de acordo com o desempenho da equipe. Quatro dimensões compõem a avaliação: (i) alcance das metas de 13 dos 74 indicadores existentes no Termo de Compromisso de Gestão - TERCOM, verificada pela chefia imediata; (ii) auto-avaliação; (iii) avaliação da satisfação da comunidade, realizada por empresa contratada; (iv) desempenho da unidade de saúde como um todo, que contratualiza 65 indicadores com a $\mathrm{SMS}^{37}$.

Ditterich $^{38}$, em estudo apresentado em 2011, sobre a percepção dos profissionais de saúde de Curitiba em relação ao uso e aplicabilidade do TERCOM e do IDQ, concluiu que a avaliação desse modelo é positiva. Ressalta, no entanto, que seria importante aprimorar o retorno do processo de avaliação individual, realizado pelas respectivas chefias aos profissionais de saúde, assim como a avaliação da comunidade atendida pelas equipes de saúde, de modo a tornar mais adequado o sistema de incentivos.

No município do Rio de Janeiro também se aplica um sistema de incentivo ao bom desempenho das equipes. Para a $\mathrm{SMS}^{39}$, a experiência portuguesa tem permitido tirar conclusões positivas 
na aplicação desta estratégia, tendo-se verificado uma melhoria dos resultados no que se refere aos indicadores contratualizados, servindo de base conceitual para a metodologia de pagamento variável apresentada neste contrato de gestão (p. 05).

São três os níveis de incentivo implementados: (i) incentivo institucional à gestão da OSS; (ii) incentivo institucional às unidades de saúde e relacionado ao alcance de objetivos, metas e indicadores de acesso, desempenho assistencial, satisfação dos usuários e eficiência; (iii) incentivos financeiros aos profissionais de saúde e relacionados à gestão da clínica e à vigilância de determinadas condições ou agravos.

\section{Discussão}

Impulsionados pelas Reformas de Estado e a Nova Gestão Pública na década de noventa, Brasil e Portugal introduzem, em processos distintos, a contratualização na atenção hospitalar e, na década seguinte, na atenção primária.

Esta pesquisa identificou diversos estudos avaliativos sobre Portugal e Curitiba, que evidenciaram a contribuição positiva da lógica contratual na coordenação dessas organizações e na melhoria de seu desempenho. Em que pese haver estudos sobre a atuação das OSS no âmbito hospitalar, seu desempenho, na estratégia saúde da família, carece de estudos analíticos e investigações com metodologias e referenciais teóricas adaptadas a essa outra realidade social da organização dos serviços e das políticas públicas no Brasil.

Para o Ministério da Saúde do Brasil, os Contratos de Gestão de Curitiba são um exemplo de boas práticas de humanização na atenção e gestão do SUS. Também considera que ocorreram mudanças positivas nos processos de trabalho, com melhor apropriação do planejamento, acompanhamento de indicadores, estratificação do risco, protocolos, perfil epidemiológico e gestão por resultados ${ }^{40}$. Para Marty ${ }^{41}$, houve reforço no compromisso das equipes, com atribuições mais claras e incremento da ação multiprofissional. Ele observou que, ao longo do processo, melhoraram os indicadores locais de saúde e o desempenho dos serviços, com as equipes realizando discussões de redirecionamento do processo do cuidado e qualidade do manejo clínico, em uma postura dos profissionais proativa e orientada a resultados.

Em Portugal, estudos evidenciaram a satisfação dos profissionais com o processo de contratualização ${ }^{42}$ e a melhoria do desempenho das $\mathrm{USF}^{43,44}$. Embora não se possa isolar a dimensão "contratualização" das demais que compõem a dinâmica das USF, esta pode ser considerada como determinante do seu desempenho, pois permitiria estabelecer os níveis de autonomia organizacional que a prestação adequada dos cuidados primários requer $^{45}$. Como exemplo, em pesquisa realizada com coordenadores de USF do país, 73,6\% responderam que a contratualização era um estímulo à qualidade do trabalho ${ }^{46}$.

Ao analisar as experiências portuguesa e brasileira, este estudo identificou tanto semelhanças no compartilhamento de problemas quanto diferenças, ambas relacionadas às especificidades de cada formação social, bem como ao conjunto de práticas que precisariam ser aperfeiçoadas para a contratualização alcançar os objetivos desejados.

O primeiro refere-se à própria introdução da contratualização no setor saúde. Ela não precisaria, necessariamente, estar associada às políticas de desestatização dos serviços, com a contratação de entes públicos não estatais ou privados. E, na perspectiva da regulação do Estado, a relação contratual público-público seria uma oportunidade de aprendizado que permitiria o desenvolvimento institucional adequado, quando da parceria com o setor privado. Nessa linha de raciocínio, Sakellarides ${ }^{47}$ afirma que o princípio do bom senso deveria fazer com que o financiador não se aventurasse excessivamente na complexa contratualização público-privado (e de implicações irreversíveis) sem adquirir uma boa experiência na contratualização público-público.

$\mathrm{O}$ segundo problema relaciona-se à própria complexidade do processo de contratualização que se caracteriza por um conjunto de procedimentos em um ambiente com sistemas de informação robustos e reestruturação organizacional interna: identificação de necessidades; estabelecimento de prioridades; verificação da capacidade instalada; negociação, fixação de objetivos e metas, estabelecimento de acordos; acompanhamento e avaliação; e aplicação de incentivos e penalizações ${ }^{21}$.

De acordo com Ferreira et al. ${ }^{9}$, o processo de contratualização na APS, torna-se ainda mais exigente na medida em que conceitos como "necessidades"; "capacidade instalada", "fixação de objetivos", "resultados" são pouco tangíveis pelo reconhecimento da dimensão subjetiva do cuidado em saúde. Soma-se a isso o fato de que a produção de cuidados em saúde, em suas várias dimensões, não define linearmente o resultado, ou seja, os ganhos em saúde.

Em um ambiente com essa complexidade, surgem obstáculos no processo que precisariam ser reconhecidos e ultrapassados sob o risco da 
contratualização deixar de ser uma lógica de gestão e tornar-se, numa visão burocratizada, estática e desarticulada do processo de avaliação de desempenho, apenas um instrumento de cobrança no cumprimento de metas. Isto reduziria seu potencial transformador e provocaria o retorno à gestão de comando e controle hierárquica.

Sabe-se que o conjunto de indicadores de monitoramento do contrato de gestão e aqueles relacionados ao sistema de incentivos necessitam de ajustes permanentes. Por um lado, apesar da contratualização ser uma estratégia de gestão por resultados, os objetivos e indicadores referemse mais aos processos do que aos resultados em saúde, ou seja, são atrelados à capacidade instalada de prestação de cuidados, ao desempenho econômico-financeiro e à eficiência. Por outro lado, metas pouco ambiciosas não premiariam o bom desempenho; e metas muito elevadas ou com indicadores voltados para uma pequena dimensão do processo de trabalho poderiam provocar quer a desmotivação nos profissionais, quer a focalização das práticas. Nesse sentido, Melo e Sousa ${ }^{48}$ afirmam que uma das consequências possíveis é aquilo a que poderemos chamar de Medicina Baseada em Indicadores, em contraponto à Medicina Centrada no Doente.

Mesmo com essas limitações, o monitoramento sistemático das metas deveria ser valorizado, pois permite analisar a tendência de cada indicador ao longo do tempo e em grupos populacionais. E mais ainda, a comparação entre equipes permitiria a troca de experiências e o aprendizado de melhores práticas.

Outros aspectos que precisariam ser aprimorados pelos entes financiadores são o fortalecimento dos mecanismos de auditoria e dos sistemas de informação e do processo de pactuação compartilhada. Cabe destacar que, enquanto no âmbito hospitalar a relação entre o financiamento e os resultados compõe o foco da lógica contratual, na APS o exercício do planejamento compartilhado entre gestores e profissionais deveria ser central, pois a proximidade com os usuários seria essencial na complicada tarefa de definição e escolha de indicadores e metas que consigam medir, com alguma sensibilidade, a evolução e efetividade de práticas de promoção, prevenção e assistência nesse domínio.

Um dos elementos facilitadores desse processo é sua construção gradual, em um ambiente de aprendizagem permanente e com o protagonismo dos profissionais de saúde. Neste sentido, a transparência e a coerência da condução, por parte dos gestores ao longo do tempo, tornam-se importante, não só para o desenvolvimento institucional, mas também para a credibilidade de todo o processo e para a motivação dos profissionais.

O protagonismo dos diversos atores, bem como a aquisição de novos conhecimentos, habilidades, sistemas tecnoadministrativos, além de arranjos de governança poderiam permitir o êxito da contratualização e dar sustentação às pactuações realizadas. Trata-se de um processo dinâmico em permanente transformação, como é a própria prestação do cuidado. Não há modelo certo ou acabado, mas sim aquele que aprende com a experiência, com a qualificação e profissionalização da gestão e também com as novas abordagens voltadas para as necessidades de saúde.

Nesse contexto, Brasil e Portugal instituíram recentemente novos processos, em dimensões distintas, e que objetivam aprimorar a prestação do cuidado em saúde pela via contratual. São, respectivamente, o Programa de Monitoramento da Qualidade e do Acesso na Atenção Básica na dimensão do desenvolvimento institucional, e o Programa de Gestão Integrada da Doença, na dimensão da integração dos cuidados na rede de serviços.

O primeiro foi criado em 2011 pelo Ministério da Saúde, com a finalidade de induzir o aprimoramento da capacidade de gestão nos níveis federal, estadual e municipal. Por meio da contratualização, por adesão espontânea, das equipes de saúde da família e vinculação do repasse de recursos federais à implantação e alcance dos padrões de acesso e de qualidade na APS, pretende promover um ambiente favorável à reflexão, aprendizagem, desenvolvimento, experimentação e inovação.

No dizer de Pinto et al. ${ }^{49}$, a adesão espontânea e contratualização individualizada por equipe introduz um dispositivo concreto de viabilização do reconhecimento intersubjetivo dos indivíduos que devem se responsabilizar pelo conjunto de ações capazes de promover a ampliação do acesso e da qualidade da atenção básica.

Cerca de 70\% dos municípios e 53,9\% das equipes de saúde da família aderiram ao primeiro ciclo de avaliação (2011/2012) e, no segundo ciclo (2013/2014), 94\% dos municípios aderiram. Além do processo em si, essa indução pode indiretamente estimular outros movimentos no nível municipal. Citam-se os exemplos das cidades de Vitória e Florianópolis que, a partir do PMAQ, instituíram incentivos financeiros vinculados ao desempenho dos profissionais de saúde que alcançassem as metas e os indicadores estabelecidos.

Em Portugal, encontra-se em desenvolvimento o modelo de Gestão Integrada da Doença ${ }^{50}$, cujo objetivo é integrar a gestão clínica de determina- 
da patologia crônica à organização da linha de cuidado em todos os níveis assistenciais, com um financiamento baseado nos resultados. Trata-se de uma abordagem de contratualização que produzirá mais conhecimento sobre a medição de resultados e custos ao longo de uma linha de cuidado, fortalecendo o processo de contratualização na perspectiva da integralidade do cuidado em saúde.

\section{Considerações finais}

As experiências aqui discutidas podem servir como fonte de aprendizagem para o refinamento de modelos mais adequados de gestão da atenção primária em cada contexto. O desafio seria identificar as oportunidades de produção de valores e princípios à cultura organizacional, que promovessem a ruptura com os padrões hierárquicos de comando e controle, e que orientassem o desempenho dos profissionais e da organização para a efetividade das ações de saúde.

\section{Colaboradores}

V Costa e Silva contribuiu na concepção da pesquisa, elaboração e redação inicial do artigo. A Escoval colaborou na revisão crítica do artigo. VA Hortale colaborou na revisão crítica do artigo e na sua revisão final.

\section{Agradecimentos}

Trabalho desenvolvido com o apoio do Programa Doutorado Sanduíche no Exterior da Coordenação de Aperfeiçoamento de Pessoal de Nível Superior do Ministério da Educação, Brasil. Bolsista da CAPES. 


\section{Referências}

1. Barzelay M. The new public management: improving research and policy dialogue. Berkeley: University of California Press; 2001.

2. Abrucio F. O impacto do modelo gerencial na administração pública: um breve estudo sobre a experiência internacional recente. Brasília: ENAP; 1996. Texto para discussão 10.

3. Trosa S. Gestão pública por resultados: quando o Estado se compromete. Rio de Janeiro, Brasilia: Renavan, ENAP; 2001.

4. Przeworski A. Sobre o desenho do Estado: uma perspectiva agent $x$ principal. In: Bresser Pereira LC, Spink PK, organizadores. Reforma do Estado e administração pública gerencial. Rio de Janeiro: Editora FGV; 1998. p. 39-73.

5. Melo MA. Governance e reforma do Estado: o paradigma agente X principal. Revista do Serviço Público 1996; 47(1).

6. Barzelay M. Origins of the new public management: an international view from public administration/political science. In: McLaughlin K, Osborne SP, Ferlie E, editors. The new public management: current trends and future prospects. London: Routledge; 2002. p. 15-33.

7. Oszlak O. From smaller to better government: the challenge of the second and third generations of state reform (internet). International Journal of Organization Theory and Behavior 2006; [acessado 12/nov/2012]; vol. IX, n. 3: Fall: United States of America. Disponível em: http:// www.oscaroszlak.org.ar/articulos-ing.php

8. Pollit C, Bouckaert G. Public Management Reform: a comparative analysis. $2^{\text {a }}$ ed. New York: Oxford University Press; 2004.

9. Ferreira AS, Escoval A, Lourenço A, Matos T, Ribeiro R. A contratualização de cuidados de saúde. In: Simões J, organizador. 30 anos do Serviço Nacional de Saúde: um percurso comentado. Coimbra: Edições Almedina SA; 2010. p. 426-460.

10. Figueras J, Robinson RJ, Jakubowski E, editors. Purchasing to improve health systems performance. Maidenhead: European Observatory on Health Systems and Policies Series, Open University Press; 2005.

11. Mintzberg H. Criando organizações eficazes: estruturas em cinco configurações. São Paulo: Atlas; 1995.

12. Figueras J, Saltman RB, Sakellarides C. Introduction. In: Saltman RB, Figueras J, Sakellarides C, editors. Critical challenges for health care reform in Europe. Buckingham: Open University Press; 1998. p. 1-21.

13. Macinko J, Starfield B, Shi L. The Contribution of Primary Care Systems to Health Outcomes within Organization for Economic Cooperation and Development (OECD) Countries, 1970-1998. Health Serv Res 2003; 38(3):831-865.

14. Rocha PM; Sá AB. Reforma da Saúde Familiar em Portugal: avaliação da implantação. Cien Saude Colet 2011; 16(6):2853-2863.

15. Portugal. Ministério da Saúde. Cuidados de Saúde Primários em Portugal. Ponto de Situação das USF. [acessado 2013 out 07]. Disponível em: http://www. acss.min-saude.pt/DepartamentoseUnidades/Unidade PlaneOrganiza\%C3\%A7\%C3\%A3odeServi\%C3\%A7osdeSa\%C3\%BAde/CuidadosdeSa\%C3\%BAdePrim\% C3\%A1rios/tabid/767/language/pt-PT/Default.aspx-
16. Pisco L. Reforma da Atenção Primária em Portugal em duplo movimento: unidades assistenciais autónomas de saúde familiar e gestão em agrupamentos de Centros de Saúde. Cien Saude Colet 2011; 16(6):2841-2852.

17. Conceição C, Fronteira I, Hipólito F, Lerberghe WV, Ferrinho P. Os grupos Alfa e a adesão ao Regime Remuneratório Experimental. Rev Port Clin Geral 2005. 21:45-59.

18. Gouveia M, Silva NS, Oliveira P, Miguel LS. Análise dos Custos dos Centros de Saúde e do Regime Remuneratório Experimental. Lisboa: Grupo de Trabalho da Associação Portuguesa de Economia da Saúde. Documento não datado [acessado 2013 jan 10]. Disponível em: http:// www.portaldasaude.pt/NR/rdonlyres/4B0B71BD1620-4168-BB98-60134AB16A49/0/Relat\%C3\%B3rio APESRRE.pdf

19. Portugal. Gabinete do Secretário de Estado Adjunto e da Saúde. Despacho normativo n. ${ }^{\circ}$ 5/2011. Diário da República 2011; 15 mar.

20. Portugal. Gabinete do Secretário de Estado Adjunto do Ministro da Saúde. Despacho no 12876/2012. Diário da República 2012; 1 out.

21. Escoval A, Matos T, Ribeiro R, Santos ATL, Oliveira MI, Cardoso MS, Jesuíno JC. Contratualização em cuidados de saúde primários: horizonte 2015/20: fase 5: relatório final. Lisboa: Administração Central do Sistema de Saúde e Escola Nacional de Saúde Pública/UNL; 2010. [acessado 2013 jan 10]. Disponível em: http://www.acss. min-saude.pt/Projectos/tabid/tabid/57/xmmid/436/ xmid/1178/xmview/2/Default.aspx

22. Brasil. Ministério da Saúde (MS). Secretaria de Atenção a Saúde. Departamento de Atenção Básica. Evolução do credenciamento e implantação da estratégia Saúde da Família. [acessado 2013 out 07]. Disponível em: http:// dab.saude.gov.br/historico_cobertura_sf.php

23. Azevedo JCR. Contrato interno de gestão e plano operativo anual da Secretaria Municipal da Saúde de Curitiba, Paraná: a percepção dos gestores locais e trabalhadores das equipes em saúde [dissertação]. Porto Alegre: Universidade Federal do Rio Grande do Sul; 2009

24. Brasil. Ministério da Administração Federal e Reforma do Estado (Mare). Organizações Sociais. Brasília: Mare; 1997. Cadernos MARE da reforma do estado; v. 2.

25. Barbosa NB, Elias PEM. As Organizações Sociais de Saúde como forma de gestão público/privado. Cien Saude Colet 2010; 15(5):2483-2495.

26. Rio de Janeiro. Decreto 30.780, de 2 de junho de 2009. Regulamenta a Lei Municipal n 5026, de 19 de Maio de 2009, que dispõe sobre a qualificação de entidades como organizações sociais e dá outras providências. Diário Oficial do Rio 2009; 3 jun.

27. Prefeitura do Rio de Janeiro. Secretaria Municipal de Saúde e Defesa Civil. Contratos de Gestão e Convênios Saúde da Família. [acessado 2013 jan 8]. Disponível em: http://www.rio.rj.gov.br/web/smsdc/exibeconteudo? article-id $=2247881$.

28. Portugal. Ministério da Saúde. Administração Central do Sistema de Saúde. Unidade Operacional de Financiamento e Contratualização (UOFC). Cuidados de saúde primários: metodologia de contratualização 2013. [acessado 2013 jun 15]. Disponível em: http://www.acss. min-saude.pt/Publicações/CuidadosdeSaúdePrimários/ tabid/118/language/pt-PT/Default.aspx 
29. Portugal. Serviço Nacional de Saúde (SNS). Grupo consultivo para a reforma dos cuidados de saúde primários. Acontecimento extraordinário: SNS: proximidade com qualidade. 2009 [acessado $2012 \mathrm{dez}$ 05]. Disponível em: http://www.portaldasaude.pt/NR/rdonlyres/7BC7CBA8-CAFB-4BA0-8CEE-D214AF7316A5/0/RelatorioCSPFev2009FECHADOx300dpi.pdf

30. Alcantara CM. A reforma gerencial e os novos modelos de gestão em saúde pública no Brasil. XVII Congreso Internacional del CLAD sobre la Reforma del Estado y de la Administración Pública, Cartagena, Colombia, 30 oct. - 2 Nov. Documento Libre. 2012

31. Di Pietro MSZ. Direito Administrativo. 25ª ed. São Paulo: Atlas; 2012.

32. Rosenthal MB, Fernandopulle R, Song HSR, Landon B. Paying For Quality: Providers' Incentives For Quality Improvement. Health Affairs 2004, 23(2):127-141.

33. England. National Health Service. Quality and Outcomes Framework for General Practice: Online GP practice results database. [acessado 2013 jan 10]. Disponível em: http:// www.qof.ic.nhs.uk

34. Kazandjian VA. Pay-for-performance in health care: the natural evolution of performance measurement and community expectations. Rev Portuguesa de Saúde Pública 2010; 9(Vol. Tem.):117-128.

35. Portugal. Ministérios das Finanças e da Administração Pública e da Saúde. Portaria n. ${ }^{\circ}$ 301/2008 de 18 de abril. Diário da República 2008; 18 abr.

36. Gouveia M, Silva NS, Oliveira P, Miguel LS. Análise dos Custos e Sistema de Remuneração e Incentivos das Unidades de Saúde Familiar. Lisboa: Grupo de Trabalho da Associação Portuguesa de Economia da Saúde; 2007.

37. Ducci L. Curitiba firma contrato de gestão com metas para a saúde. Rev Brasileira Saúde da Família. Brasilia 2007; 14:43-57.

38. Ditterich RG. A contratualização como ferramenta de gestão e organização da atenção primária à saúde: avanços, dificuldades e desafios [tese]. Curitiba: Pontifícia Universidade Católica do Paraná; 2011.

39. Rio de janeiro. Secretaria Municipal de Saúde e Defesa Civil. Anexo B do Contrato de Gestão: acompanhamento do contrato, avaliação e metas para os serviços de saúde [acessado 2013 jan 28]. Disponível em: http://subpav. com.br/download/prot/Pagamento_por_desempenho _novas_metas_2011_2012.pdf.

40. Brasil. Ministério da Saúde (MS). Secretaria de Atenção à Saúde. Política Nacional de Humanização. Boas práticas de humanização na atenção e gestão do Sistema Único de Saúde - SUS: contrato interno de gestão na Secretaria Municipal de Saúde de Curitiba. Brasília: MS; 2006.

41. Marty IK. Primeiras experiências de contratos de gestão em Curitiba: Secretaria Municipal da Saúde. In: Giacomini CH, organizador. Gestão para resultados em Curitiba: a experiência de contratualização. Curitiba: Instituto Municipal de Administração Pública; 2009. p. 43-56.
42. Ferreira PL, Antunes P. Monitorização da satisfação dos profissionais das USF. Coimbra: CEISUC, 2009. [acessado 2013 jan 20]. Disponível em: http://www.mcsp. min-saude.pt/Imgs/content/page_56/us_prof_rel.pdf.

43. Ferreira PL, Antunes P, Portugal S. O valor dos cuidados primários: perspectiva dos utilizadores das USF-2009. Lisboa: VFBM Comunicação Ltda.; 2010.

44. Rocha PM, Sá AB. Reforma da Saúde Familiar em Portugal: avaliação da implantação. Cien Saude Colet 2011; 16(6):2853-2863.

45. Portugal. Serviço Nacional de Saúde (SNS). Grupo consultivo para a reforma dos cuidados de saúde primários. Acontecimento extraordinário: proximidade com qualidade. 2009 [acessado $2012 \mathrm{dez}$ 05]. Disponível em: http://www.portaldasaude.pt/NR/rdonlyres/7BC 7CBA8-CAFB-4BA0-8CEE-D214AF7316A5/0/Relatorio CSPFev2009FECHADOx300dpi.pdf

46. Biscaia AR, Vilas Boas B, Cardeira R, Vieira CP. O Momento Actual da Reforma dos Cuidados de Saúde Primários em Portugal 2011/2012 - Relatório de estudo. Lisboa: Associação Nacional de Unidades de Saúde Familiar; 2012.

47. Sakellarides C. Repensando a contratualização. Revista Portuguesa de Saúde Pública 2010; 9(Vol. Tem.):5-6.

48. Melo M, Sousa JC. Os Indicadores de Desempenho Contratualizados com as USF: Um ponto da situação no actual momento da Reforma. Rev Port Clin Geral 2011; 27(1):28-34.

49. Pinto HA, Sousa A, Florêncio AR. O Programa Nacional de Melhoria do Acesso e da Qualidade da Atenção Básica: Reflexões sobre o seu desenho e processo de implantação. RECIIS - R. Eletr. de Com. Inf. Inov. Saúde [periódico na Internet]. 2012 Ago [acessado 2013 jan 30]; 6(Supl. 2): [cerca de 19 p.]. Disponível em: http://www.reciis.cict. fiocruz.br/index.php/reciis/article/view/624.

50. Escoval A, Coelho A, Diniz JA, Rodrigues M, Moreira F, Espiga P. Gestão integrada da doença: uma abordagem experimental de gestão em saúde. Revista Portuguesa de Saúde Pública 2010; 9(Vol. Tem.):105-116.

Artigo apresentado em 15/07/2013

Aprovado em 29/09/2013

Versão final apresentada em 07/10/2013 